\title{
Hepatitis $C$ bio-behavioural surveys in people who inject drugs - a systematic review of sensitivity to the theoretical assumptions of respondent driven sampling
}

\author{
Ryan Buchanan ${ }^{1 *}$, Salim I. Khakoo², Jonathan Coad², Leonie Grellier ${ }^{3}$ and Julie Parkes ${ }^{1}$
}

\begin{abstract}
Background: New, more effective and better-tolerated therapies for hepatitis $\mathrm{C}$ (HCV) have made the elimination of HCV a feasible objective. However, for this to be achieved, it is necessary to have a detailed understanding of HCV epidemiology in people who inject drugs (PWID).

Respondent-driven sampling (RDS) can provide prevalence estimates in hidden populations such as PWID. The aims of this systematic review are to identify published studies that use RDS in PWID to measure the prevalence of HCV, and compare each study against the STROBE-RDS checklist to assess their sensitivity to the theoretical assumptions underlying RDS.
\end{abstract}

Method: Searches were undertaken in accordance with PRISMA systematic review guidelines. Included studies were English language publications in peer-reviewed journals, which reported the use of RDS to recruit PWID to an HCV bio-behavioural survey. Data was extracted under three headings: (1) survey overview, (2) survey outcomes, and (3) reporting against selected STROBE-RDS criteria.

Results: Thirty-one studies met the inclusion criteria. They varied in scale (range 1-15 survey sites) and the sample sizes achieved (range 81-1000 per survey site) but were consistent in describing the use of standard RDS methods including: seeds, coupons and recruitment incentives.

Twenty-seven studies (87\%) either calculated or reported the intention to calculate population prevalence estimates for HCV and two used RDS data to calculate the total population size of PWID. Detailed operational and analytical procedures and reporting against selected criteria from the STROBE-RDS checklist varied between studies. There were widespread indications that sampling did not meet the assumptions underlying RDS, which led to two studies being unable to report an estimated HCV population prevalence in at least one survey location.

Conclusion: RDS can be used to estimate a population prevalence of HCV in PWID and estimate the PWID population size. Accordingly, as a single instrument, it is a useful tool for guiding HCV elimination. However, future studies should report the operational conduct of each survey in accordance with the STROBE-RDS checklist to indicate sensitivity to the theoretical assumptions underlying the method.

Systematic review registration: PROSPERO CRD42015019245

Keywords: Hepatitis C, Prevalence, Respondent-driven sampling, Intravenous, Drug

\footnotetext{
* Correspondence: ryan.buchanan@nhs.net

'Department of Population Science and Medical Statistics, Faculty of

Medicine, University of Southampton, C level, South Academic block,

Southampton, Hampshire, UK

Full list of author information is available at the end of the article
} 


\section{Background}

Hepatitis C (HCV) infects over 150 million people and alongside other causes of viral hepatitis is a leading cause of death worldwide [1, 2]. New, more effective and better-tolerated treatments for $\mathrm{HCV}$ are now widely available; however, the accurate epidemiological data required to guide the logistical and financial planning needed for disease elimination strategies is lacking [3].

Over ten million people worldwide who currently inject drugs (PWID) and many more with a past history of injecting drug use are thought to be chronically infected with HCV [4], and it is the treatment of PWID rather than other patients with $\mathrm{HCV}$ that is likely to have the most profound impact on $\mathrm{HCV}$ prevalence [5]. However, it is within this population that there are particular concerns about the validity of epidemiological estimates as these are generally based on data from convenience samples in 'easy to reach' PWID $[4,6]$.

PWID are hidden by social stigma and the illegality of their practice, and therefore, it is difficult to obtain a representative sample necessary to make population prevalence estimates [7]. Interest and experience in studying hard-to-reach or hidden populations developed substantially in the wake of the HIV epidemic in the 1990s. At this time, the difficultly of obtaining representative samples with existing survey techniques prompted the development of a method called respondent-driven sampling (RDS) [8].

RDS begins with a sample of seeds from the target population who are keen to participate in the survey and usually socially well connected. The seeds are then asked to refer a pre-defined number, or 'quota', of contacts to the survey who form wave 1 of recruitment; these responders are then asked to refer wave 2 and so on. In this way, a sample with maximal recruitment, i.e. a full quota of new recruits in each wave, expands geometrically. Recruitment throughout the waves is driven by a primary incentive for taking part and usually a secondary incentive for recruiting others [8].

Harnessing social influence through the use of incentives gives RDS the potential to reach participants who would not normally come forward to a researcher, and the limited recruitment quota (usually three) minimises selection bias for those with large social networks [8]. This allows the characteristics of a sample to reach a steady state or 'equilibrium' quickly-often after just four waves of recruitment [8]. In addition, specific software has been developed which incorporates estimators to calculate prevalence estimates for the entire target population from data collected during the sampling process $[9,10]$.

However, these estimators rely on methodological assumptions, which relate to the underlying size and network structure of the target population and participant recruitment behaviour $[11,12]$.

Previous reviews of RDS in HIV bio-behavioural surveys $[13,14]$ have highlighted concerns about the quality of reporting and led, in 2015, to the publication of the Strengthening the Reporting of Observational Studies in Epidemiology for respondent-driven sampling studies (STROBE-RDS) reporting checklist [15]. This document aims to improve the quality of reporting and includes 22 items that outline how studies should report survey data collected using RDS. Importantly, it incorporates criteria that indicate sensitivity to the assumptions underlying the population estimates.

Whilst the use of RDS in HIV epidemiology has been the subject of several systematic reviews, its use in the investigation of $\mathrm{HCV}$ epidemiology and specifically the sensitivity of prevalence estimates to the assumptions of RDS is not described [13, 14]. The aims of this systematic review are to identify published studies documenting the use of RDS in HCV bio-behavioural surveys of PWID and describe the sensitivity of population estimates to the theoretical assumptions of RDS. To do so, the reported operational and analytical conduct of each study is compared against selected criteria from the STROBE-RDS checklist [15].

\section{Method}

The systematic review protocol was published on the PROSPERO website under registration number CRD42015019245 prior to commencing the literature search, and the review was conducted according to the PRISMA statement [16, 17].

\section{Information sources and literature search}

Two scoping searches were initially undertaken using MEDLINE in March 2015 with no date or language limitations. The first used the terms 'PWID" or IDU" or Injecting drug user* AND Hepatitis $\mathrm{C}$ or $\mathrm{HCV}$ AND respondent driven sampl"'. On title and abstract review, this identified 14 potentially eligible studies and was compared to a second scoping search for the term 'respondent driven sampl ${ }^{*}$; this identified three additional studies suggesting the initial search was too specific.

Accordingly in the final search, MEDLINE, SCOPUS and WEB of SCIENCE online databases were searched, with no language or date limitations for the broad term 'respondent driven sampl*' between the 10 April 2015 to 31 December 2016. This was followed by a forward and backward citation search in the SCOPUS database and a manual citation search through selected papers.

Further searching was conducted through 'grey literature' sources including institution and key author websites, which included Respondentdrivensampling.org (Cornell University) and lisagjohnston.com. Specific 
search phrases in these domains varied but reflected the inclusion and exclusion criteria. Two experts with experience undertaking surveys and teaching in this field were also contacted and asked to comment on the included studies and suggest others that may meet the inclusion criteria.

\section{Inclusion criteria and study selection}

Peer-reviewed studies written in English were included if they:

- Reported a survey in a population of PWID

- Reported the use of RDS as the sampling method

- Reported a sample prevalence or an estimated population prevalence for $\mathrm{HCV}$

As HCV can remain asymptomatic and therefore undiagnosed for many decades after infection, 'PWID' was interpreted as anyone who had ever injected drugs [18]. Studies using mixed survey methods (for example, combined convenience sampling and RDS) and not reporting results separately were excluded. Non-English language papers were not eligible for inclusion as translation services were beyond the resources of this review; however, this was deliberately not a specific search criterion so the quantity of otherwise eligible non-English literature could be assessed.

Duplicate studies were removed from selected titles and abstracts in Mendeley reference manager. Two researchers (RB and JC), experienced in systematic review methodology, independently assessed the selected titles and abstracts for inclusion using a selection tool and resolved discrepancies by discussion with a third researcher (JP). The full papers of selected abstracts were obtained and subject to further independent review for inclusion. Where two studies reported data from the same survey and both published HCV prevalence, the study that was published first was included.

\section{Data extraction}

Data was extracted under three headings: (1) survey overview, (2) survey outcomes and (3) reporting against selected STROBE-RDS criteria.

$\mathrm{RB}$ and JC extracted the data independently, and where referenced, additional papers describing the survey method in more detail were accessed and further details recorded.

\section{Results}

\section{Search results}

The initial search of the online databases identified 4060 titles; of these, 1815 were duplicates leaving 2245 separate studies. Abstract and title review identified 50 studies potentially meeting the inclusion criteria. Citation, grey literature searches and expert recommendation identified a further ten studies for full paper review (Fig. 1). Sixty studies were obtained and reviewed in full. A further 29 were excluded at this stage with 31 remaining that met the inclusion criteria; Fig. 1 outlines the specific reasons for exclusion.

\section{Overview of included surveys}

Included studies were published between 2006 and 2016 and reported either a sample or population prevalence of HCV in PWID. They included surveys from Europe, North America, Asia, the Middle East, Africa and Australasia.

Eighteen studies (58\%) conducted RDS in a single target population although this varied with the largest taking place in 15 cities across India [19]. Of the studies reporting from multiple locations, two used overlapping data from the same survey $[20,21]$ and one study included survey sites that did not use RDS [22].

All studies clearly defined their eligibility criteria for participation and reported how the sample prevalence of HCV was obtained (Table 1). Fifteen surveys (48\%) reported how participants were followed up by the research



Fig. 1 Flow diagram of studies screened and assessed for inclusion. Specific reasons for exclusion are indicated 


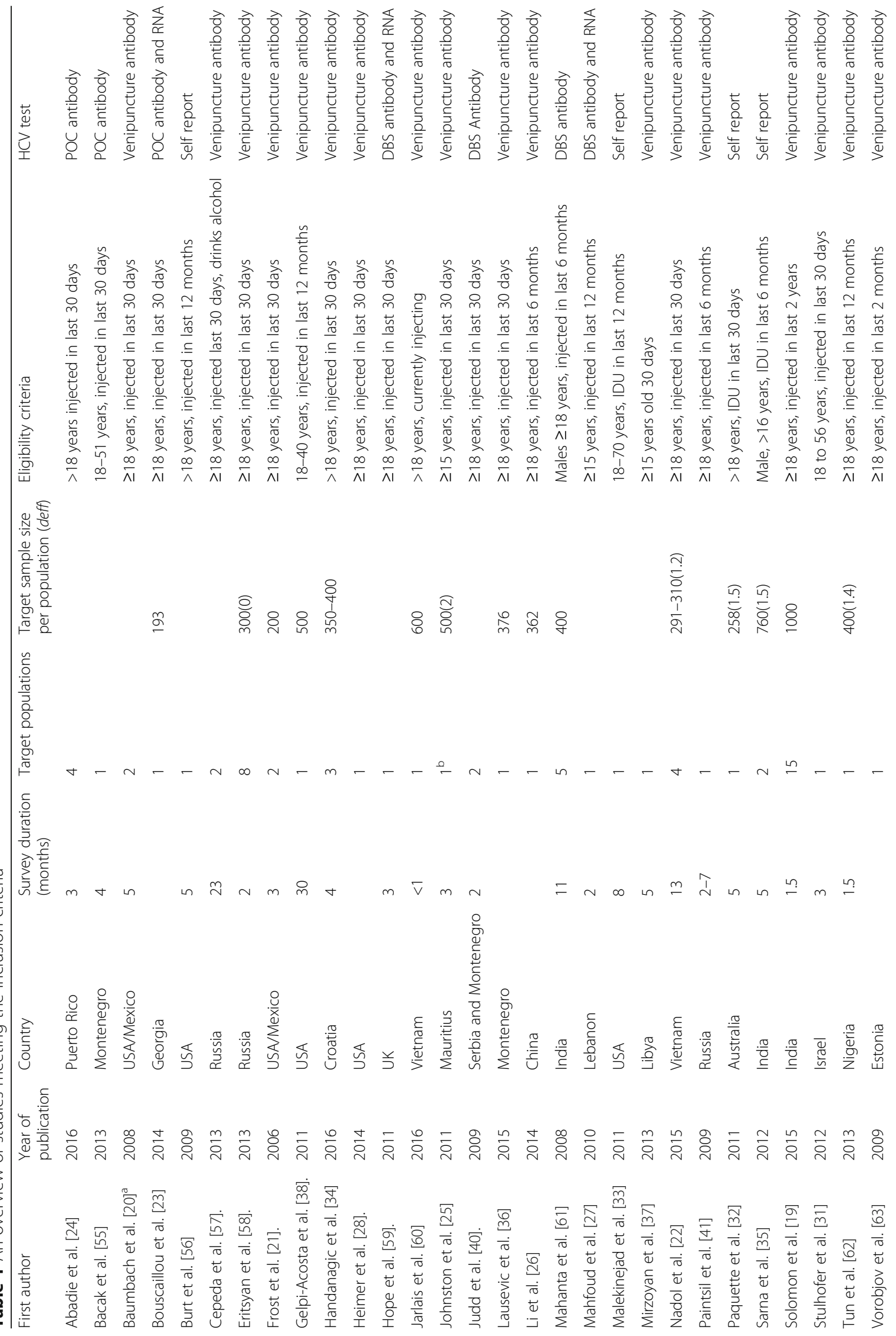




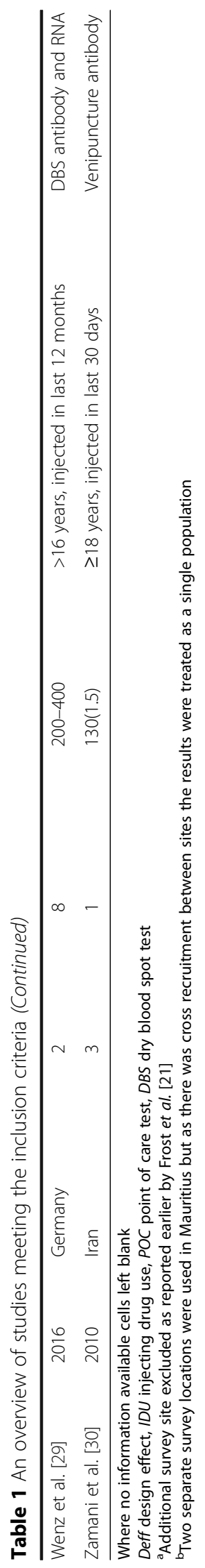


team, in most of these participants were advised to collect their testing results and were traced back to these via a 'linked anonymous record, i.e. the participant retained a unique identifier that connected them to their blood sample. However, two studies actually reported incentivising participants to return to collect their results [20, 21], three described a direct referral pathway from the research team to specialist services $[20,23,24]$ and one of these also took the opportunity to give out harm reduction advice and, where necessary, vaccination against hepatitis A and hepatitis B [20].

Twenty studies (65\%) reported the time taken to reach the final sample size, and seventeen studies (55\%) documented a target sample size although of these, only seven reported the value of the design effect (deff) used in making the calculation (Table 1). The final sample size at each survey site was reported in most studies (97\%) (mean 382; range 81-1000), and in accordance with the inclusion criteria, all the selected papers published either the sample HCV prevalence or a population prevalence estimate.

Two studies (6\%) went on to use sampling data in combination with 'service multipliers' to calculate a total population size of PWID and therefore gave an indication of the total disease burden of $\mathrm{HCV}$ in the target population for the survey $[25,26]$.

\section{Sensitivity to RDS assumptions}

Four studies deliberately treated their survey data as a convenience sample and did not report any intention to calculate population estimates. The remaining 27 studies either calculated or reported the intention to calculate population prevalence estimates, and these studies were therefore constrained by the theoretical assumptions underlying RDS.

Table 2 gives an overview of reporting in these studies against selected STROBE-RDS criteria that give an indication about sensitivity and adherence to these assumptions. The following section describes how these 27 studies reported against these criteria and where there was evidence that the assumptions were not met.

\section{Assumption 1: participant social networks are linked into a single component}

There were indications given in three studies (11\%) that the underlying network structure adversely affected recruitment $[20,27,28]$ and from a single study that clustering within the network affected the validity of population prevalence estimates [29]. These studies did not describe formative research to explore the structure of the social network in advance of the survey, but this was described in nine other studies. Among these, there was variation in the scale and methods used; some studies reported the use of informal interviews with local stakeholders, whilst others described focus groups, qualitative interviews and ethnography or cited a published preliminary study. Only one study specifically described how this formative work was used to optimise recruitment from all parts of the network [30].

\section{Assumption 2: recruiters do not pass coupons to strangers and ties are reciprocal}

Two studies (7\%) reported a number of participants being recruited to the survey by strangers, but neither described how these participants were handled in the analysis $[30,31]$. Overall, sixteen studies (59\%) reported the recording of the relationship between the recruiter and recruit; however, only one precisely defined the question that was used to assess this [32].

\section{Assumption 3: estimates are independent of seed characteristics}

Eight studies (30\%) reported the purposive selection of seeds through ethnography or via consultation with key stakeholders in the field. Nineteen studies (70\%) described the number of seeds used to initiate recruitment (range 2 to 82) although only two met the STROBE-RDS checklist by describing clearly how many seeds were added to boost recruitment after the survey had started [33, 34]. The data from one survey could not be used to calculate a population prevalence for $\mathrm{HCV}$ because too many seeds had been needed to reach the target sample size [28].

The recruitment quota, or number of coupons given to each seed, was reported in all studies and ranged from 2 to 4 , but the number of recruitment waves per seed was poorly described with only three studies including diagrammatic recruitment 'trees' within the main text [21, 29, 33]. However, 15 studies (56\%) reported the number of waves achieved in the longest recruitment chain (range 5-50), and another reported a median chain length across 15 survey sites [19]. Seven studies (26\%) reported measuring 'sampling equilibrium' after a certain number of recruitment waves for key criteria to indicate independence of the sample from seed characteristics and one used this as the point to stop sampling [26].

How seed data was handled in the analysis was not explicitly reported in most studies although six (22\%) did describe deliberately excluding seed data from population prevalence estimates whereas two (7\%) specifically documented its inclusion [25, 29].

\section{Assumption 4: recruiters pass coupons randomly to eligible network members and these individuals are equally likely to participate}

One study clearly described how participants were trained to recruit social network members to the survey [33], but there were concerns expressed in a number of 


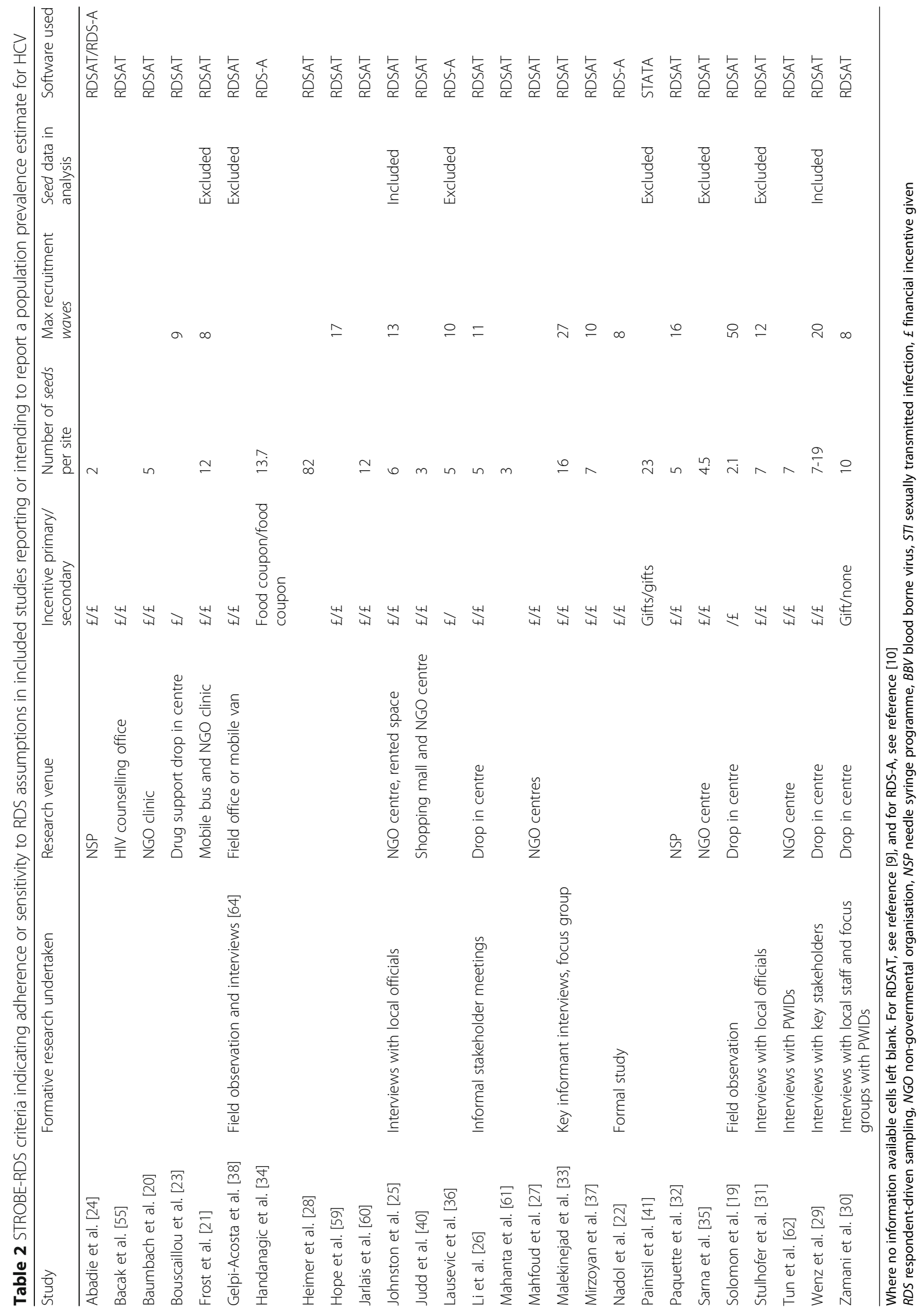


studies about non-random recruitment. Eight studies (30\%) reported difficulty recruiting female participants despite, in one, the deliberate use of female seeds [23]. One study considered whether this was a true representation of the underlying population structure [35], but three expressed concern about 'response bias' attributed to cultural barriers within the target population $[23,36,37]$. Other studies expressed concerns about the non-recruitment of participants from particular ethnic backgrounds [32], socio-economic groups [28] or geographical areas [32, 33]. To test recruitment bias, three studies (11\%) reported measuring homophily for selected characteristics between recruits and recruiters $[24,29,34]$ and in one homophily between persons with a known HCV-positive status was recorded [24].

Sixteen studies (59\%) described the venue used for the survey, and one reported concerns that the venue may have influenced participation [34]. The incentives used for recruiting others to the survey were also described in 21 studies (78\%), and 19 of these described a financial primary and secondary incentive, the value for which ranged from $\$ 50$ and $\$ 20$, respectively, in the USA [38] and $\$ 1$ and $\$ 0.8$ in India $[19,35]$. Where reported, the remaining surveys used gifts or food coupons [30,34,39]. A single survey expressed concern that the financial incentive may have led to bias towards poorer PWID and one did not use a secondary incentive for this reason [30]. Another study was concerned that the offer (as part of participation) of being linked directly to $\mathrm{HCV}$ care may have encouraged a disproportionate number of PWID with HCV to attend [23].

\section{Assumption 5: participants only take part once and are eligible members of the target population}

A single study described participants attempting to attend more than once and non-eligible individuals trying to take part [40]. The method used to screen survey participants for eligibility (i.e. proof they had injected drugs) was recorded in 15 studies (56\%), but only four described how repeat attenders were identified. Of these, a single study recorded identifiers such as tattoos or anthropometric measurements [41] and one used finger print records [19].

\section{Assumption 6: participants accurately report their degree size}

Fifteen studies (56\%) reported recording the degree size for each recruit, and of these, three precisely described the question or questions used to define this [20, 29, 33]. No studies reported testing the sensitivity of prevalence estimates against variations in degree size.

\section{Assumption 7: sampling occurs with replacement}

The majority (85\%) of included studies used a version of RDSAT software [9] to calculate prevalence estimates. RDSAT incorporates an estimator that is constrained by this assumption [11, 42, 43]; however, just a single study measured how this may have affected the HCV prevalence estimate by comparing it against an estimate calculated with a successive sampling estimator [24].

\section{Assumption 8: an estimate of total target population size is known in advance of the survey}

Four studies (15\%) used a successive sampling estimator integrated within RDSanalyst software [10] to calculate population estimates and therefore needed a target population size estimate to make the calculation. Two specifically reported the use of such an estimate and referenced its source $[22,34]$.

\section{Discussion}

The studies included in this review used RDS to recruit over 25,000 PWID to bio-behavioural surveys across five continents. The studies were consistent in documenting the use of standard RDS methods including recruitment coupons, recruitment quotas and incentives to facilitate the coupon exchange but varied considerably in scale, duration and operational conduct.

The quality of reporting against the STROBE-RDS criteria, in some instances, made an assessment about the sensitivity of survey results to the underlying assumptions of RDS difficult. The incomplete reporting of the sampling method in surveys using RDS has been described before $[13,14]$ and is not surprising here given that the STROBE-RDS checklist was published after most of the included studies [15]. Nevertheless, from what was reported, there were indications that the assumptions were not met in some studies, and in two cases, this led to study authors being unable to use survey data to calculate a population prevalence estimate. This is consistent with reports elsewhere which describe recruitment via non-reciprocal relationships [44], inaccurate degree size reporting [45], biased recruitment according to ethnicity [46] and limited recruitment due to disparate social networks within the target population [47].

The collective understanding of the implications of not meeting the assumptions of RDS has advanced in recent years through literature 'testing the assumptions' [45, 48-51]. Simulation studies have reported the scale of biases associated with seeds, recruitment waves, high recruitment homophily and sampling without replacement [50], whilst work based on real-world surveys has demonstrated the bias associated with inaccurate reporting of degree size [45]. This has led to the evolution of the original RDS estimator [11, 42, 52], new estimators 
based on successive sampling and ego network data [53, 54], and development of RDS technical procedur$\mathrm{e}$-an iterative temporal transformation that may account for some of the variation seen in the included studies.

Specifically, this has led to development in how to accurately ascertain degree size, how to handle seed data in the analysis [50] (a contrast with earlier literature [11]), how to measure sample independence from seed characteristics using convergence rather than equilibrium [12], and the use of ego network data to assess recruitment bias (65).

This systematic review is the first to describe the use of RDS in HCV epidemiology and explore sensitivity to the methodological assumptions underlying RDS in these studies. In so doing, it draws attention to reporting criteria for surveys using RDS and highlights recent technical developments. However, it also has areas of potential bias, for example, the search strategy, by including only peer-reviewed publications, excluded survey data within grey literature such as public health reports. This may have led to bias towards the more successful, robustly designed surveys that have a higher chance of publication. In so doing, this review may have overestimated the quality of reporting relating to the assumptions of RDS and underestimated sensitivity to these assumptions.

\section{Conclusion}

RDS can improve our understanding of $\mathrm{HCV}$ epidemiology in PWID and therefore has the potential to make an important contribution to the global elimination strategy for HCV. This robust systematic review included 31 studies and showed that operational procedures varied between studies and were frequently incompletely reported. There were also widespread indications of sensitivity to the methodological assumptions of RDS that, in some studies, prevented the estimation of $\mathrm{HCV}$ population prevalence. Future surveys using RDS to explore the epidemiology of $\mathrm{HCV}$ within PWID should convey sensitivity to the assumptions by reporting in accordance with the STROBE-RDS checklist and should consider using recent advances in the procedural and analytical methods of RDS.

\section{Abbreviations}

Deff: Design effect; HCV: Hepatitis C; PWID: People who inject drugs; RDS: Respondent-driven sampling

\section{Acknowledgements}

Thanks to Lisa G Johnston and Matthew Hickman for reading an early draft of the study and suggesting additional studies that should also be considered for inclusion. Additional thanks also to study authors who responded by email to questions about the operational conduct of each survey.
Funding

RB has a fellowship award with the NIHR (National Institute of Health Research) CLAHRC scheme.

\section{Availability of data and materials}

The dataset supporting the conclusions of this study is available as part of the Open Science framework in the repository https://osf.io/s8cgv/.

\section{Authors' contributions}

All authors were involved in the development of the design of the study. RB undertook the literature search, and JC and RB reviewed the selected titles and abstracts and then full papers for inclusion. JP was consulted in the event of disagreement. RB and JC independently undertook the data extraction, and all authors contributed to the drafting of the study and have read and approved the final manuscript.

Ethics approval and consent to participate

Not applicable.

\section{Consent for publication}

Not applicable.

\section{Competing interests}

The authors declare that they have no competing interests.

\section{Publisher's Note}

Springer Nature remains neutral with regard to jurisdictional claims in published maps and institutional affiliations.

\section{Author details}

${ }^{1}$ Department of Population Science and Medical Statistics, Faculty of Medicine, University of Southampton, C level, South Academic block, Southampton, Hampshire, UK. ${ }^{2}$ Faculty of Medicine, University of

Southampton, E level, South Academic block, Southampton, Hampshire, UK. ${ }^{3}$ Department of Gastroenterology, St Mary's Hospital, Isle of Wight, Newport, UK.

Received: 18 April 2017 Accepted: 25 June 2017

Published online: 11 July 2017

\section{References}

1. Mohd Hanafiah K, Groeger J, Flaxman AD, Wiersma ST. Global epidemiology of hepatitis C virus infection: new estimates of age-specific antibody to HCV seroprevalence. Hepatology. 2013;57(4):1333-42.

2. Stanaway JD, Flaxman AD, Naghavi M, Fitzmaurice C, Vos T, Abubakar I, et al. The global burden of viral hepatitis from 1990 to 2013: findings from the Global Burden of Disease Study 2013. Lancet. Elsevier; 2016;388(10049):98897.

3. Wiktor SZ, Hutin YJ-F, WHO, Collaborators G 2013 M and C of D, Mahajan R, Xing J, et al. The global burden of viral hepatitis: better estimates to guide hepatitis elimination efforts. Lancet. Elsevier; 2016;388(10049):117-71.

4. Nelson PK, Mathers BM, Cowie B, Hagan H, Des Jarlais D, Horyniak D, et al. Global epidemiology of hepatitis B and hepatitis $C$ in people who inject drugs: results of systematic reviews. Lancet (London, England). 2011; 378(9791):571-83.

5. Innes H, Goldberg D, Dillon J, Hutchinson SJ. Strategies for the treatment of hepatitis $C$ in an era of interferon-free therapies: what public health outcomes do we value most? Gut. BMJ Publishing Group Ltd and British Society of Gastroenterology; 2015;64(11):1800-9.

6. Public Health England. Unlinked anonymous monitoring survey of people who inject drugs in contact with specialist services. 2014. Available from: https://www.gov.uk/government/statistics/people-who-inject-drugs-hiv-andviral-hepatitis-monitoring.

7. Magnani R, Sabin K, Saidel T, Heckathorn D. Review of sampling hard-to-reach and hidden populations for HIV surveillance. AIDS. 2005; 19 Suppl 2:S67-72.

8. Heckathorn D. Respondent-driven sampling: a new approach to the study of hidden populations. Social problems. 1997:44(2):174-99.

9. Volz E, Wejnert C, Cameron C, Spiller M, Barash V, Degani I, et al. Respondent-driven sampling analysis tool (RDSAT) version 7.1. Ithaca NY: Cornell Univeristy; 2012. 
10. Handcock MS, Fellows I, Gile K. RDS analyst: software for the analysis of respondent-driven sampling data, version 0.51. 2014.

11. Salganik MMJ, Heckathorn DDD. Sampling and estimation in hidden populations using respondent-driven sampling. Sociol Methodol. 2004;34(1): 193-239.

12. Gile KJ, Johnston LG, Salganik MJ. Diagnostics for respondent-driven sampling. J R Stat Soc Ser A (Statistics Soc. Blackwell Publishing Ltd; 2015; 178(1):241-69.

13. Montealegre JR, Johnston LG, Murrill C, Monterroso E. Respondent driven sampling for HIV biological and behavioral surveillance in Latin America and the Caribbean. AIDS Behav. 2013;17(7):2313-40.

14. Malekinejad M, Johnston LG, Kendall C, Kerr LRFS, Rifkin MR, Rutherford GW. Using respondent-driven sampling methodology for HIV biological and behavioral surveillance in international settings: a systematic review. AIDS Behav. 2008:12(4 Suppl):S105-30.

15. White R, Hakim A, Salganik M, Spiller M, Johnston L, Kerr L, et al. Strengthening the reporting of observational studies in epidemiology for respondent-driven sampling studies: "STROBE-RDS" statement. J Clin Epidemiol. Elsevier; 2015;68(12):1463-71.

16. Buchanan R, Coad J, Parkes J, Khakoo S, Grellier L. PROSPERO international prospective register of systematic reviews respondent driven sampling methodology for estimating hepatitis $C$ epidemiology in people who inject drugs: a systematic review. 2015. Available from: http://www.crd.york.ac.uk/ PROSPERO/DisplayPDF.php?ID=CRD42015019245.

17. Moher D, Liberati A, Tetzlaff J, Altman DG. Preferred reporting items for systematic reviews and meta-analyses: the PRISMA statement. PLoS Med. 2009;6(7):e1000097.

18. Hoofnagle $\mathrm{JH}$. Hepatitis C: The clinical spectrum of disease. Hepatology. Wiley Subscription Services, Inc., A Wiley Company; 1997;26(S3):15S-20S.

19. Solomon SS, Mehta SH, SriKrishnan AK, McFall AM, Laeyendecker O, Celentano DD, et al. Burden of hepatitis $C$ virus disease and access to hepatitis C virus services in people who inject drugs in India: a crosssectional study. Lancet Infect Dis. Lancet Publishing Group; 2015;15(1): 36-45.

20. Baumbach JP, Foster LN, Mueller M, Cruz MF, Arbona S, Melville S, et al. Seroprevalence of select bloodborne pathogens and associated risk behaviors among injection drug users in the Paso del Norte region of the United States-Mexico border. Harm Reduct J. 2008:5:33.

21. Frost SDW, Brouwer KC, Firestone Cruz MA, Ramos R, Ramos ME, Lozada RM, et al. Respondent-driven sampling of injection drug users in two U.S.-Mexico border cities: recruitment dynamics and impact on estimates of HIV and syphilis prevalence. J Urban Health. 2006;83(6 Suppl):i83-97.

22. Nadol P, O'connor S, Duong H, Le L-VN, Thang PH, Tram TH, et al. Findings from integrated behavioral and biologic survey among males who inject drugs (MWID) - Vietnam, 2009-2010: evidence of the need for an integrated response to HIV, hepatitis B virus, and hepatitis C virus. PLoS One. Public Library of Science; 2015;10(2):e0118304.

23. Bouscaillou J, Champagnat J, Luhmann N, Avril E, Inaridze I, Miollany V, et al. Hepatitis C among people who inject drugs in Tbilisi, Georgia: an urgent need for prevention and treatment. Int J Drug Policy. Elsevier; 2014:25(5):871-8.

24. Abadie R, Welch-Lazoritz M, Gelpi-Acosta C, Reyes JC, Dombrowski K. Understanding differences in HIV/HCV prevalence according to differentiated risk behaviors in a sample of PWID in rural Puerto Rico. Harm Reduct J. 2016;13(1):10

25. Johnston L, Saumtally A, Corceal S, Mahadoo I, Oodally F, Johnston L, et al. High HIV and hepatitis $C$ prevalence amongst injecting drug users in Mauritius: findings from a population size estimation and respondent driven sampling survey. Int J Drug Policy. 2011;22(4):252-8.

26. Li L, Assanangkornchai S, Duo L, McNeil E, Li J. Risk behaviors, prevalence of HIV and hepatitis $C$ virus infection and population size of current injection drug users in a China-Myanmar Border City: results from a respondentdriven sampling survey in 2012. PLoS One. 2014;9(9):e106899.

27. Mahfoud Z, Afifi R, Ramia S, El Khoury D, Kassak K, El Barbir F, et al. HIV/AIDS among female sex workers, injecting drug users and men who have sex with men in Lebanon: results of the first biobehavioral surveys. AIDS. 2010; 24 Suppl 2(SUPPL. 2):S45-54.

28. Heimer R, Barbour R, Palacios WR, Nichols LG, Grau LE, Heimer R, et al. Associations between injection risk and community disadvantage among suburban injection drug users in southwestern Connecticut, USA. AIDS Behav. Springer New York LLC; 2014;18(3):452-63.
29. Wenz B, Nielsen S, Gassowski M, Santos-Hövener C, Cai W, Ross RS, et al. High variability of HIV and HCV seroprevalence and risk behaviours among people who inject drugs: results from a cross-sectional study using respondent-driven sampling in eight German cities (2011-14). BMC Public Health. 2016;16(1):927.

30. Zamani S, Radfar R, Nematollahi P, Fadaie R, Meshkati M, Mortazavi S, et al. Prevalence of HIV/HCV/HBV infections and drug-related risk behaviours amongst IDUs recruited through peer-driven sampling in Iran. Int J Drug Policy. 2010;21(6):493-500

31. Stulhofer A, Chetty A, Rabie RA, Jwehan I, Ramlawi A. The prevalence of HIV $\mathrm{HBV}, \mathrm{HCV}$, and HIV-related risk-taking behaviors among Palestinian injecting drug users in the East Jerusalem Governorate. [Erratum appears in J Urban Health. 2012:89(4):677; PMID: 22782840]. J Urban Heal. 2012;89(4):671-6.

32. Paquette DM, Bryant J, De Wit J, Paquette DM, Bryant J, De Wit J. Use of respondent-driven sampling to enhance understanding of injecting networks: a study of people who inject drugs in Sydney, Australia. Int J Drug Policy. 2011;22(4):267-73.

33. Malekinejad M, McFarland W, Vaudrey J, Raymond HF, Malekinejad M, McFarland W, et al. Accessing a diverse sample of injection drug users in San Francisco through respondent-driven sampling. Drug Alcohol Depend. 2011;118(2-3):83-91.

34. Handanagic S, Bozicevic I, Civljak M, Dominkovic Z, Sevic S, Barbaric J, et al. HIV and hepatitis C prevalence, and related risk behaviours among people who inject drugs in three cities in Croatia: Findings from respondentdriv..._-PubMed_NCBI. Int J Drug Policy. 2016;32:57-63.

35. Sarna A, Tun W, Bhattacharya A, Lewis D, Singh YS, Apicella L, et al. Assessment of unsafe injection practices and sexual behaviors among male injecting drug users in two urban cities of India using respondent. Southeast Asian J Trop Med Public Heal. 2012;43(3):652-67.

36. Lausevic D, Begic S, Mugosa B, Terzic N, Vratnica Z, Labovic I, et al. Prevalence of HIV and other infections and correlates of needle and syringe sharing among people who inject drugs in Podgorica, Montenegro: a respondent-driven sampling survey. Harm Reduct J. BioMed Central Ltd.; 2015;12(2):2

37. Mirzoyan L, Berendes S, Jeffery C, Thomson J, Ben Othman H, Danon L, et al. New evidence on the HIV epidemic in Libya: why countries must implement prevention programs among people who inject drugs. J Acquir Immune Defic Syndr JAIDS. 2013;62(5):577-83.

38. Gelpí-Acosta C, Hagan H, Jenness SM, Wendel T, Neaigus A. Sexual and injection-related risks in Puerto Rican-born injection drug users living in New York City: a mixed-methods analysis. Harm Reduct J. 2011;8:28.

39. Paintsil E, Verevochkin SV, Dukhovlinova E, Niccolai L, Barbour R, White E, et al. Hepatitis C virus infection among drug injectors in St Petersburg, Russia: social and molecular epidemiology of an endemic infection. Addiction. 1881;104(11):1881-90

40. Judd A, Rhodes T, Johnston LG, Platt L, Andjelkovic V, Simić D, et al. Improving survey methods in sero-epidemiological studies of injecting drug users: a case example of two cross sectional surveys in Serbia and Montenegro. BMC Infect Dis. 2009;9:14.

41. Paintsil E, Verevochkin SV, Dukhovlinova E, Niccolai L, Barbour R, White E, et al. Hepatitis C virus infection among drug injectors in St Petersburg, Russia: social and molecular epidemiology of an endemic infection. Addiction. 2009:104(11):1881-90.

42. Volz E, Heckathorn, Douglas D. Probability based estimation theory for respondent driven sampling. J Off Stat. 2008;24(1):79-97.

43. Heckathorn DD. Extensions of respondent-driven sampling: analyzing continuous variables and controlling for differential recruitment. Sociol Methodol. 2007;37(1):151-207.

44. Lansky A, Drake A, Wejnert C, Pham H, Cribbin M, Heckathorn DD. Assessing the assumptions of respondent-driven sampling in the national HIV behavioral surveillance system among injecting drug users. Open AIDS J. 2012;6(SPEC.ISSUE 1):77-82.

45. Mills HL, Johnson S, Hickman M, Jones NS, Colijn C. Errors in reported degrees and respondent driven sampling: implications for bias. Drug Alcohol Depend. Elsevier Ireland Ltd; 2014;142:120-6.

46. Wang J, Falck RS, Li L, Rahman A, Carlson RG. Respondent-driven sampling in the recruitment of illicit stimulant drug users in a rural setting: findings and technical issues. Addict Behav. 2007;32(5):924-37.

47. Bryant J. Using respondent-driven sampling with "hard to reach" marginalised young people: problems with slow recruitment and small network size. Int J Soc Res Methodol. Routledge; 2013;17(6):599-611. 
48. Rudolph AE, Young AM, Lewis CF. Assessing the geographic coverage and spatial clustering of illicit drug users recruited through respondent-driven sampling in New York City. J Urban Health. Springer Science and Business Media Deutschland GmbH; 2015;92(2):352-78.

49. Rudolph AE, Fuller CM, Latkin C, Rudolph AE, Fuller CM, Latkin C, et al. The importance of measuring and accounting for potential biases in respondent-driven samples. AIDS Behav. 2013;17(6):2244-52.

50. Gile KJ, Handcock MS. Respondent-driven sampling: an assessment of current methodology. Sociol Methodol. 2010;40(1):285-327.

51. Gile KJ, Johnston LG, Salganik MJ. Diagnostics for respondent-driven sampling. StartFragmentJournal of the Royal Statistical Society: Series A (Statistics in Society). EndFragment2012; 178(1):241-269.

52. Heckathorn DD, Semaan S, Broadhead RS, Hughes JJ. Extensions of respondent-driven sampling: a new approach to the study of injection drug users aged 18-25. AIDS Behav. 2002;6(1):55-67.

53. Gile KJ. Improved inference for respondent-driven sampling data with application to HIV prevalence estimation. J Am Stat Assoc. 2011;106(493):135-46.

54. Lu X. Linked ego networks: improving estimate reliability and validity with respondent-driven sampling. Soc Networks. 2013;35(4):669-85.

55. Baćak V, Laušević D, Mugoša B, Vratnica Z, Terzić N, Bacak V, et al. Hepatitis $C$ virus infection and related risk factors among injection drug users in Montenegro. Eur Addict Res. 2013;19(2):68-73.

56. Burt RD, Thiede H, Hagan H, Burt RD, Thiede H, Hagan H. Serosorting for hepatitis $C$ status in the sharing of injection equipment among Seattle area injection drug users. Drug Alcohol Depend. 2009;105(3):215-20.

57. Cepeda JA, Niccolai LM, Eritsyan K, Heimer R, Levina O, Cepeda JA, et al. Moderate/heavy alcohol use and HCV infection among injection drug users in two Russian cities. Drug Alcohol Depend. 2013;132(3):571-9.

58. Eritsyan K, Heimer R, Barbour R, Odinokova V, White E, Rusakova MM, et al. Individual-level, network-level and city-level factors associated with HIV prevalence among people who inject drugs in eight Russian cities: a crosssectional study. BMJ Open. 2013;3(6):1-11.

59. Hope VD, Hickman M, Ngui SL, Jones S, Telfer M, Bizzarri M, et al. Measuring the incidence, prevalence and genetic relatedness of hepatitis $C$ infections among a community recruited sample of injecting. J Viral Hepat. 2011;18(4): 262-70.

60. Des Jarlais D, Duong HT, Pham Minh K, Khuat OHT, Nham TTT, Arasteh K, et al. Integrated respondent-driven sampling and peer support for persons who inject drugs in Haiphong, Vietnam: a case study with implications for interventions. AIDS Care. Taylor \& Francis; 2016;28(10):1312-5.

61. Mahanta J, Medhi GK, Paranjape RS, Roy N, Kohli A, Akoijam BS, et al. Injecting and sexual risk behaviours, sexually transmitted infections and HIV prevalence in injecting drug users in three states in India. AIDS. 2008;22 Suppl 5:S59-68.

62. Tun W, Vu L, Adebajo SB, Abiodun L, Sheehy M, Karlyn A, et al. Populationbased prevalence of hepatitis $B$ and $C$ virus, HIV, syphilis, gonorrhoea and chlamydia in male injection drug users in Lagos, Nigeria. Int J STD AIDS. 2013:24(8):619-25.

63. Vorobjov S, Des Jarlais DC, Abel-Ollo K, Talu A, Ruutel K, Uuskula A. Sociodemographic factors, health risks and harms associated with early initiation of injection among people who inject drugs in Tallinn, Estonia: evidence from cross-sectional surveys. Int J Drug Policy. 2013;24(2):150-5.

64. Allen DR, Finlayson T, Abdul-Quader A, Lansky A. The role of formative research in the national HIV behavioral surveillance system. Public Health Rep. 2009. 124(1):26-33.

\section{Submit your next manuscript to BioMed Central and we will help you at every step:}

- We accept pre-submission inquiries

- Our selector tool helps you to find the most relevant journal

- We provide round the clock customer support

- Convenient online submission

- Thorough peer review

- Inclusion in PubMed and all major indexing services

- Maximum visibility for your research

Submit your manuscript at www.biomedcentral.com/submit 